\title{
Spinal Cord Sarcoidosis: An Unusual Cause of Neurogenic Bladder Dysfunction
}

\author{
Ali Chouaib $^{\mathrm{a}, \mathrm{c}}$, Patrick Cabanis ${ }^{\mathrm{a}}$, Thierry Billebaud ${ }^{\mathrm{a}}$, Meryeme EL Machkour ${ }^{\mathrm{b}}$
}

\begin{abstract}
We report a case of spinal cord sarcoidosis in which urodynamic studies showed neurogenic bladder dysfunction. A 56-year-old Caucasian woman developed progressive weakness of both lower extremities causing walking disturbance. Two months later, she became unable to walk, and she gradually developed urinary urgency, nocturnal urinary frequency, and urge urinary incontinence. Spinal MRI disclosed dorsal lesion, with atrophy of the cervical cord; the brain MRI was normal. Cerebrospinal fluid (CSF) analysis showed elevated proteins. Serum angiotensin-converting enzyme (ACE) level was moderately high. The tuberculin skin test was negative. The thoraco-abdomino-pelvien scanner showed a suspicious lesion in the liver. The draining biopsy of the liver revealed non-caseating epithelioid granuloma. This finding confirmed the diagnosis of spinal cord sarcoidosis. The urodynamic study showed detrusor hyperreflexia. Prednisolone treatment improved the urinary and neurological symptoms. The spinal lesion seems to be responsible for the mictional disturbance in our patient with spinal cord sarcoidosis.
\end{abstract}

Keywords: Sarcoidosis; Spinal cord; Urodynamics; Neurogenic bladder

\section{Introduction}

Sarcoidosis is a multisystem inflammatory granuloma-

\footnotetext{
Manuscript accepted for publication June 8, 2011

${ }^{a}$ Department of Urology, Creteil's Inter-Communal Hospital, Creteil, France

${ }^{b}$ Department of Neurology, Kremlin-Bicetre Hospital, APHP, Paris, France

${ }^{c}$ Corresponding author: Ali Chouaib, 315, 1 Boulevard Jourdan, 75014, Paris, France. Email: alichouaib@gmail.com
}

doi:10.4021/jnr21e tous disease of unknown etiology. Neurosarcoidosis (NS), sarcoidosis involving the nervous system, is thought to be much rarer, occurring in less than $5 \%$ of patients with systemic sarcoidosis [1]. However, in a half of them, the presenting manifestation is neurologic without any evidence of systemic involvement, making early diagnosis and management difficult.

Sarcoidosis of the spinal cord is rare with an incidence estimated at $0.43 \%$ [2].

Mictional disturbance is occasionally described in patients with spinal cord sarcoidosis, but few urodynamic findings are available to our knowledge [3].

We describe a case history and the result of urodynamic study in a patient with spinal cord sarcoidosis.

\section{Case Report}

\section{Case history}

A 56-year-old Caucasian woman developed progressive weakness of both lower extremities causing walking disturbance; two months later, she became unable to walk. However, she gradually developed urinary urgency, nocturnal urinary frequency (more than five times a night), and urge urinary incontinence. Physical examination noted a spastic paraparesis, tetrapyramidal irritation and instability. Spinal MRI disclosed dorsal lesion which appeared as a low signal intensity on T1 and high signal intensity on T2 (Fig. 1), with atrophy of the cervical cord (Fig. 2). The brain MRI was normal. Cerebrospinal fluid (CSF) analysis showed elevated proteins $(0.86 \mathrm{mg} / \mathrm{L})$. Serum angiotensin-converting enzyme (ACE) level was moderately elevated. The tuberculin skin test was negative. The initial phosphocalcic balance assessment was normal but repetitive controls put in evidence and hypercalciuria $(51.85 \mathrm{~g} / \mathrm{L})$ with a hypophosphaturia. The thoraco-abdomino-pelvien computed tomography scanner showed a suspicious lesion in the liver. The draining biopsy of the liver revealed non-caseating epithelioid granuloma. This finding confirmed the diagnosis of spinal cord sarcoidosis.

The patient underwent corticoid therapy (500 mg/day 


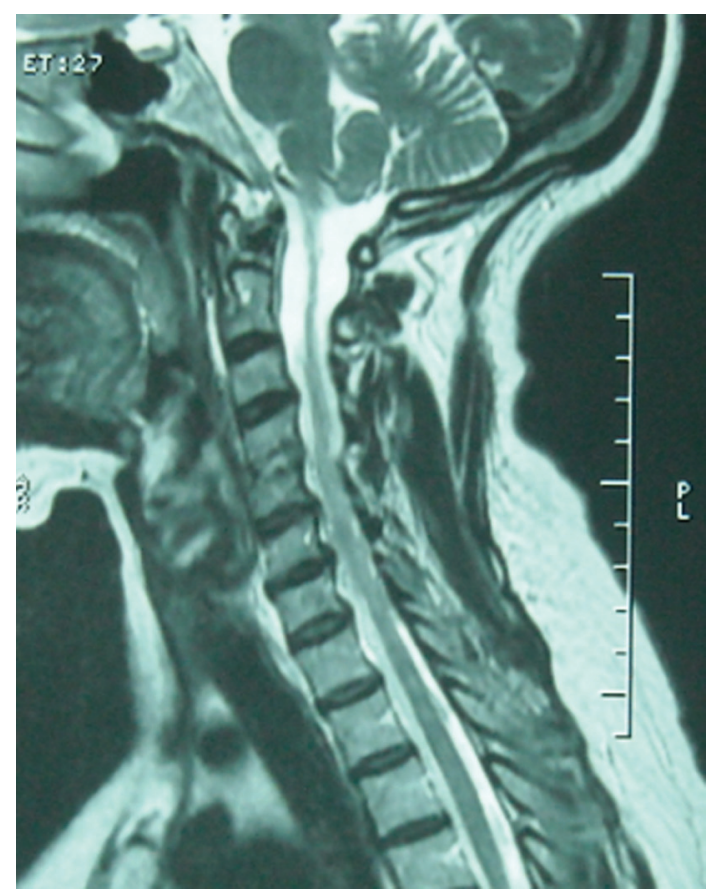

Figure 1. MRI of the spinal cord (sagittal plane), T2 weighted image. The T9 intramedullary region (dorsal spine) of the cord was high signal intensity.

of intravenous methylprednisolone over 5 succeeding days) and started taking oral prednisolone ( $40 \mathrm{mg}$ /day). After four weeks, a control MRI of the spine showed improvement. The patient continued to improve gradually and her urinary symptoms were ameliorated, and the treatment was tapered down by $10 \mathrm{mg}$ every week to a maintenance dose of $10 \mathrm{mg}$ / day. Walking became possible with two sticks.

\section{Urodynamic study findings}

Urodynamic study was done one month after the start of treatment. The urine culture was sterile and cystoscopy was normal. After voluntary voiding, we measured post-micturition residuals, and their normal volume is under $30 \mathrm{~mL}$. After inserting a 12-F side-hole catheter, we measured urethral pressure profilometry with infusing $2 \mathrm{~mL} / \mathrm{min}$ of sterile water. Normal range of maximum urethral closure pressure (UPmax) in our laboratory is $>41 \mathrm{~cm} \mathrm{H}_{2} \mathrm{O}$ but $<82 \mathrm{~cm} \mathrm{H}_{2} \mathrm{O}$. After inserting an 8-F double-lumen transurethral catheter and a concentric needle electrode, medium-fill $(50 \mathrm{~mL} / \mathrm{min})$ water cystometry with simultaneous sphincter EMG was done by a urodynamic computer (Lifetech; Janus) in a supine position. Normal range of first sensation is $>100 \mathrm{~mL}$ but $<$ $300 \mathrm{~mL}$, and bladder capacity is $>200 \mathrm{~mL}$ but $<600 \mathrm{~mL}$. The methods and definitions used for the urodynamic studies conformed to the standards proposed by the International Continence Society [Abrams et al., 1989]. The patient had no amount of post-micturition residual. External sphincter EMG revealed exaggerated bulbocavernosus reflex, but no

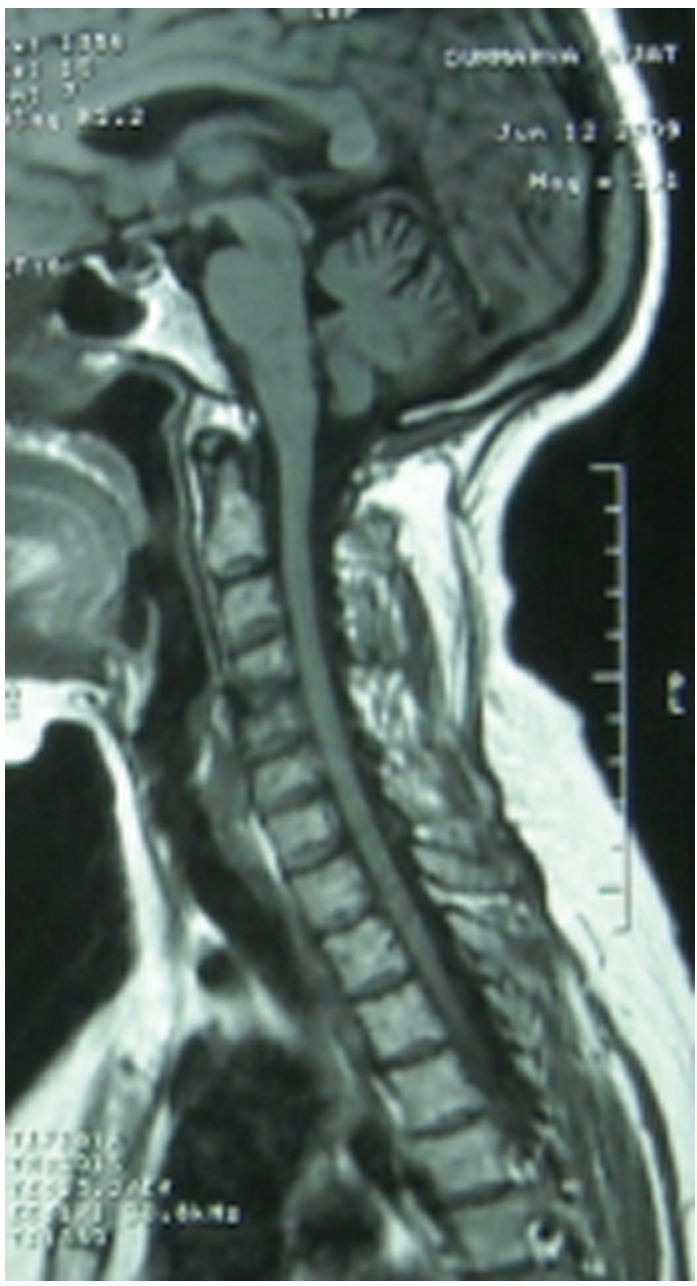

Figure 2. MRI of the spinal cord (sagittal plane), T1 weighted image. The T9 intramedullary region (dorsal spine) of the cord was low signal intensity, with atrophy of cervical cord.

evidence of uninhibited sphincter relaxation or detrusorsphincter dyssynergia. Water cystometry revealed decreased bladder volume at first sensation of $80 \mathrm{~mL}$, bladder capacity of $230 \mathrm{~mL}$, and a marked detrusor hyperreflexia (Fig. 3). The maximum urethral closure pressure was $60 \mathrm{~cm} \mathrm{H}_{2} \mathrm{O}$. Though we could not repeat urodynamic studies, the corticosteroid therapy has improved urinary symptoms.

\section{Discussion}

Sarcoidosis is a multisystem granulomatous disease of unknown etiology that most commonly affects the lungs, eyes and skin. Sarcoidosis affects the nervous system in 5\% of patients [1], being considered a rare manifestation. Sarcoidosis of the spinal cord is rare with an incidence estimated at $0.43 \%$ [2]. Like other spinal cord injuries, the spinal cord sarcoidosis may be presented with paraparesis, quadripa- 


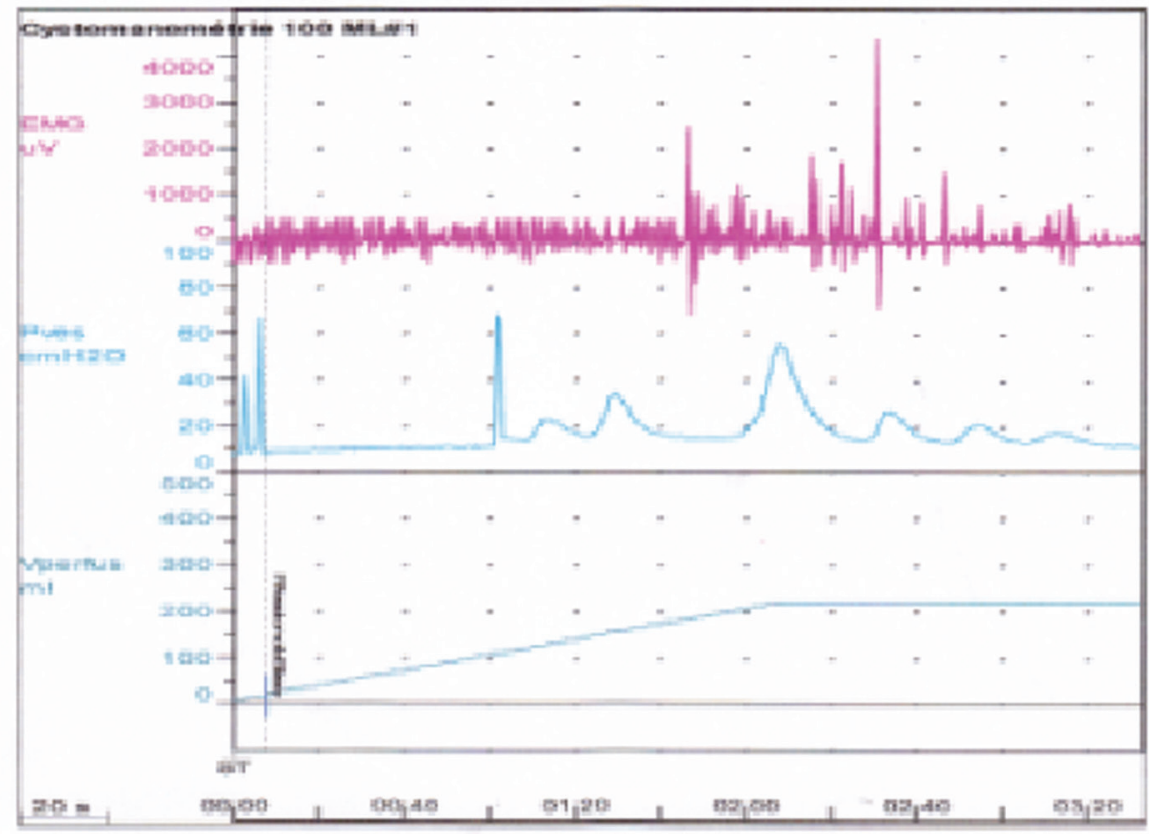

Figure 3. EMG-cystometry, involuntary detrusor contraction.

resis, sensory changes, autonomic dysreflexia, or radicular symptoms $[4,5]$.

MRI is very sensitive in localizing the lesions; lesions enhance with gadolinium except late in the disease, when cord atrophy occurs. Radiologic aspects are not specific and highly variable $[3,6]$.

The diagnosis is suspected in patients with active systemic sarcoidosis; however, it is extremely difficult to make the diagnosis when spinal cord involvement is the first or sole manifestation of the disease. Biopsy of the spinal cord is an invasive procedure and is not without risk. The decision to obtain a biopsy of the spinal cord should be taken only after an extensive search for other organ involvement [3, 6].

This case is an example of fruitful search of other organ involvement and avoidance to biopsy the spinal cord.

For the treatment of spinal cord sarcoidosis, corticosteroids are the therapy of choice.

Urinary problems caused by neurosarcoidosis are exceptional, especially for spinal cord sarcoidosis. Detailed description is rare and few urodynamic findings are available on the mictional disturbance in spinal cord sarcoidosis. In most published cases, the authors reported only the clinical description of the lower urinary tract disorders associated with neurosarcoidosis. Fitzpatrick KJ et al described the first case of neurogenic lower urinary tract dysfunction associated with spinal cord sarcoidosis [7]. Urodynamic findings of detrusor hyperreflexia with detrusor-sphincter dyssynergia correlate with magnetic resonance imaging (MRI) examination which found intramedullary involvement at the mid-thoracic level.

Sakaibara $\mathrm{R}$ et al [8] reported a 42-year-old woman who began to have a slowly progressive spastic gait, left hand numbness, urinary urgency/frequency, and voiding difficulty which worsened gradually for a year. Two months later she became unable to walk without an aid. Spinal MRI disclosed C2-7 cord swelling. She also developed bilateral hilar lymphadenopathy, uveitis, and an increased serum concentration of angiotensin-converting enzyme (ACE). Endoscopic lymph node biopsy showed non-caseating epithelioid granuloma. These findings and the clinical features confirmed the diagnosis of spinal cord sarcoidosis.

\section{Conclusion}

Spinal cord sarcoidosis is a rare but well-documented condition; however in several cases the presenting manifestation is neurologic without any evidence of systemic involvement, making early diagnosis and management difficult. The spinal lesions may be mainly responsible for mictional disturbance in patients with spinal cord sarcoidosis.

The present case and the previous reports reflect the phenotypic variability of this disorder, and the benefit to perform urodynamic studies.

\section{References}

1. Joseph FG, Scolding NJ. Sarcoidosis of the nervous system. Pract Neurol. 2007;7(4):234-244.

2. Bogousslavsky J, Hungerbuhler JP, Regli F, Graf HJ. Subacute myelopathy as the presenting manifestation of sarcoidosis. Acta Neurochir (Wien). 1982;65(3-4):193- 
197.

3. Saleh S, Saw C, Marzouk K, Sharma O. Sarcoidosis of the spinal cord: literature review and report of eight cases. J Natl Med Assoc. 2006;98(6):965-976.

4. Hamasaki T, Noda M, Kamei N, Yamamoto S, Ochi M, Yasunaga Y. Intradural extramedullary mass formation in spinal cord sarcoidosis: case report and literature review. Spine (Phila Pa 1976). 2003;28(20):E420-423.

5. Sakakibara R, Hattori T, Uchiyama T, Yamanishi T. Micturitional disturbance in a patient with neurosarcoidosis.
Neurourol Urodyn. 2000;19(3):273-277.

6. Fitzpatrick KJ, Chancellor MB, Rivas DA, Kumon H, Mandel S, Manon-Espaillat R. Urologic manifestation of spinal cord sarcoidosis. J Spinal Cord Med. 1996;19(3):201-203.

7. Sakaibara R, Uchiyama T, Kuwabara S, Kawaguchi N, Nemoto I, Nakata M, Hattori H. Autonomic dysreflexia due to neurogenic bladder dysfunction; an unusual presentation of spinal cord sarcoidosis. J Neurol Neurosurg Psychiatry. 2001;71(6):819-820. 